\title{
High-pT Jet Energy Scale Uncertainty from single hadron response with the ATLAS detector
}

\author{
Trine Poulsen*i \\ Lund University (SE) \\ E-mail: trine.poulsen@cern.ch
}

\begin{abstract}
The jet energy scale (JES) uncertainty is estimated using different methods at different $\mathrm{p}_{\mathrm{T}}$ ranges. In-situ techniques exploiting the $\mathrm{p}_{\mathrm{T}}$ balance between a jet and a reference object (e.g. Z or gamma) are used at lower $\mathrm{p}_{\mathrm{T}}$, but at very high $\mathrm{p}_{\mathrm{T}}(>2.5 \mathrm{TeV})$ there is not enough statistics for such in-situ techniques. A low JES uncertainty at high- $\mathrm{p}_{\mathrm{T}}$ is important in several searches for new phenomena, e.g. the dijet resonance and angular searches. In the highest $\mathrm{p}_{\mathrm{T}}$ range, the JES uncertainty is estimated using the calorimeter response to single hadrons. In this method, jets are treated as a superposition of energy depositions of single particles. An uncertainty is applied to each energy deposition belonging to the particles within the jet, and propagated to the final jet energy scale. This poster presents the JES uncertainty found with this method at $\operatorname{sqrt}(\mathrm{s})=8 \mathrm{TeV}$ and its developments.
\end{abstract}

Fourth Annual Large Hadron Collider Physics

13-18 June 2016

Lund, Sweden

${ }^{*}$ Speaker.

${ }^{\dagger}$ On behalf of the ATLAS Collaboration. 


\section{Introduction}

The jet energy has to be well calibrated and understood to be able to use jets for physics measurements and searches. The jet calibration has many steps [1] but in this context the most relevant are to bring the jet from the electromagnetic scale to the hadronic scale using Monte Carlo (MC) simulation and afterwards to use in-situ calibrations to account for the differences between data and MC.

The in-situ techniques exploits the $\mathrm{p}_{\mathrm{T}}$ balance between a jet and a reference object $(\mathrm{Z}, \gamma$ or multijet) but can only be used at lower $\mathrm{p}_{\mathrm{T}}$. At very high $\mathrm{p}_{\mathrm{T}}(>2.5 \mathrm{TeV})$ there are not enough events in the processes used for these balancing techniques. Here the single particle approach is needed instead.

\section{The ATLAS detector}

These studies are performed with the ATLAS detector [2], which is a multi-purpose particle detector. It has a forward-backward symmetric, cylindrical geometry and a near $4 \pi$ coverage in solid angle around the collision point. It consists of an inner tracking detector surrounded by a thin superconducting solenoid, electromagnetic and hadronic calorimeters and a muon spectrometer.

\section{Single particle approach}

In ATLAS a variety of jets are used. In this case, the used jets are found with the anti- $\mathrm{K}_{\mathrm{t}}$ algorithm from topological clusters. The algorithm groups the clusters around the hardest objects to give jets with a regular boundary shape, which is unaffected by soft radiation.

However, jets can also be seen as a collection of particles. The single particle approach associate the energy deposited by the jet with the particles using MC samples, where the true characteristics of the particles are known. Different uncertainties applying to the particles can then be convoluted into the JES uncertainty.

The method can be summarized in a few steps. Firstly, the deposited energy by every particle in the jet is identified. Then the energy is smeared by different uncertainties depending on the characteristics of the particle ( $\mathrm{p}, \mathrm{p}_{\mathrm{T}}, \eta$, charge etc.). In the end, the smeared energy of the particles is summed and compared to the unsmeared energy of the jet.

The different uncertainties, which are applied to the particles, can be grouped in several categories:

- E/p: Measurements of the ratio of the energy, E, deposited in the calorimeter to the momentum, $\mathrm{p}$, measured by the inner tracking detector are available for particles with $0.5<\mathrm{p}$ $[\mathrm{GeV}]<30$ and $|\eta|<2.3$. The measurements in data are compared to MC to give uncertainties on the response and the probability of getting tracks with no associated clusters in the calorimeter, such that $\mathrm{E} / \mathrm{p}=0$ (acceptance) [3].

- Combined Test Beam (CTB): Measurements of the barrel calorimeter response to hadrons are available for particles with $20<\mathrm{p}[\mathrm{GeV}]<350$ and $|\eta|<0.65$. As for the E/p measurement the data is compared to MC to give the uncertainties on the response. [4]. 
- Out of Range: When E/p and CTB measurements are not available a flat $10 \%$ uncertainty is applied to the particles.

- Electromagnetic scale: The uncertainties on the electromagnetic scales have been directly measured. $Z \rightarrow e e$ decays were used for the LAr calorimeter, whereas energy loss of minimum ionising muons were used in the Tile calorimeter.

- Neutral: GEANT4 studies show that an additional uncertainty of 10\% (5\%) apply to neutral particles below (above) $3 \mathrm{GeV}$. Furthermore, an uncertainty of $10 \%$ applies to the long-lived neutral kaon, $\mathrm{K}_{L}[3]$.

For $\mathrm{e}^{-}, \mathrm{e}^{+}, \gamma$ and $\pi^{0}$ only the electromagnetic scale uncertainties apply, since they lose energy electromagnetically. On the other hand the electromagnetic scale uncertainties are not applied, whenever the E/p uncertainties are, since they are already included in the form of differences in the EM scale calibration on data and MC.

\section{Results}

When the smeared energy of the particles has been summed to give the smeared energy of the jet, the response can be calculated as the linear sum of the smeared energy from the different particle uncertainties described above divided by the unsmeared energy. The effect each of the particle uncertainties will have on the jet energy can be calculated as the smeared energy from that uncertainty divided by the unsmeared energy. To get the total JES uncertainty the individual uncertainties are added in quadrature.

The result from $\sqrt{s}=7$ and $8 \mathrm{TeV}$ dataset is shown in Figure 1. Here the E/p uncertainties are divided into the E/p response and the E/p acceptance. The CTB and Out of Range uncertainties are added together in the CTB response and the electromagnetic scale uncertainties are shown as the global energy scale.

The results can also be used to evaluate the correlations in the JES uncertainty of jets in different $\left(\mathrm{p}_{\mathrm{T}}, \eta\right)$-bins. This is shown in Figure 2. It is seen that the correlation between different bins is $\sim 100 \%$ for neighbouring bins as expected, while for widely separated bins it is $\sim 30 \%$.

\section{References}

[1] ATLAS Collaboration, Monte Carlo Calibration and Combination of In-situ Measurements of Jets in ATLAS, ATL-COM-PHYS-2015-071 (2015), [https : / / cds . cern. ch/record/1987296].

[2] ATLAS Collaboration, The ATLAS Experiment at the CERN Large Hadron Collider, JINST 3 (2008) S08003.

[3] ATLAS collaboration, Single hadron response measurement and calorimeter jet energy scale uncertainty with the ATLAS detector at the LHC, The European Physical Journal C, [10.1140/epjc/s10052-013-2305-1].

[4] ATLAS collaboration, Study of energy response and resolution of the ATLAS barrel calorimeter to hadrons of energies from 20 to $350 \mathrm{GeV}$, Nuclear Instruments and Methods in Physics Research Section A, [http://dx.doi.org/10.1016/j.nima.2010.04.054]. 


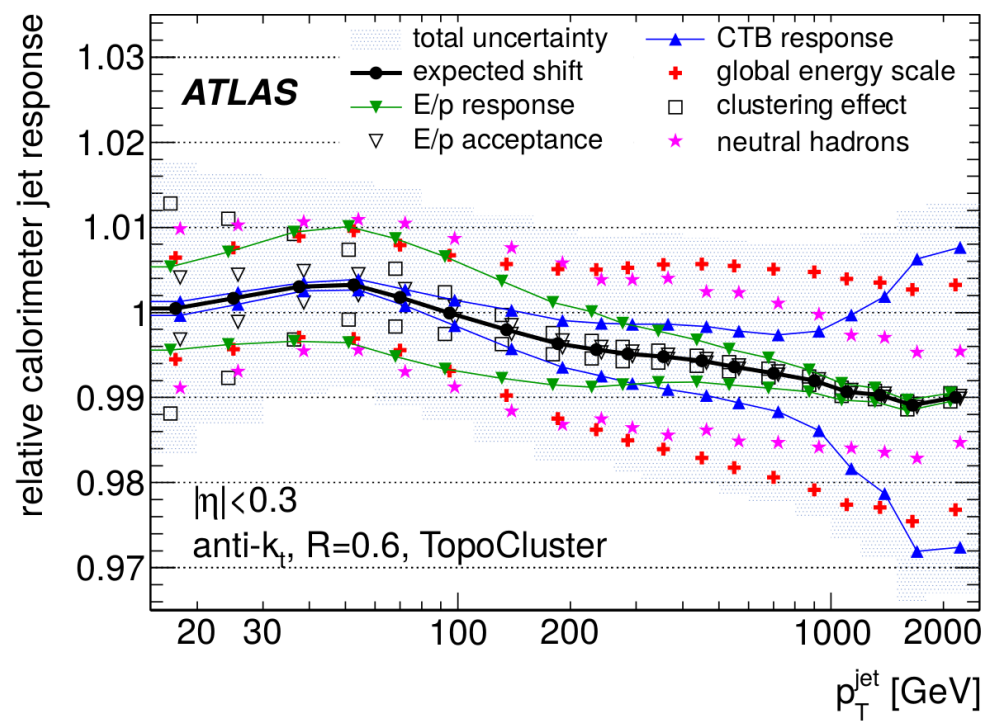

Figure 1: The jet response and uncertainty in bins of jet $\mathrm{p}_{\mathrm{T}}$ from $\sqrt{s}=7$ and $8 \mathrm{TeV}$ datasets [3].

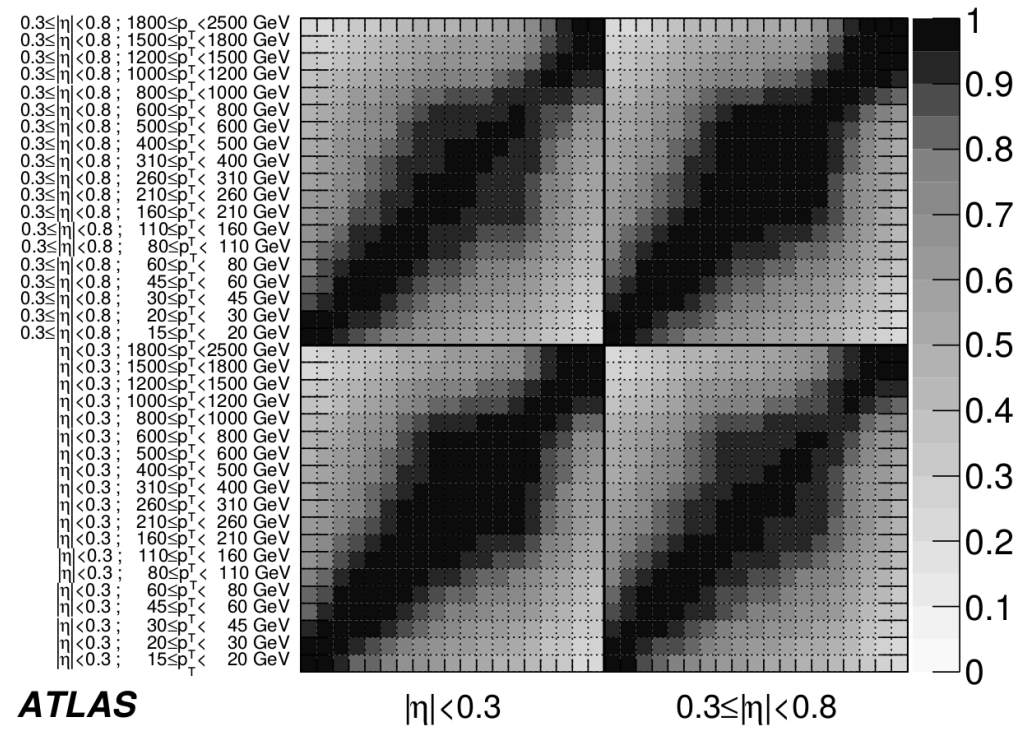

Figure 2: Correlation in JES uncertainty from $\sqrt{s}=7$ and $8 \mathrm{TeV}$ datasets [3]. 